\title{
Fatores que predispõem a quedas em idosos residentes na região oeste de Santa Maria, RS
}

Predsposing factorsfor fallsin the ddely residentsin nestem region of SantaMaria, RS

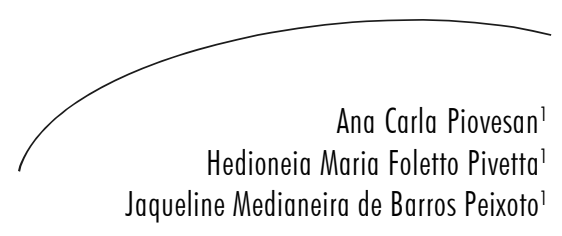

Resumo

A fisioterapia desempenha importante papel na prevenção das quedas na pessoa idosa. Os objetivos deste estudo foram investigar os fatores de risco que predispõem a quedas em idosos residentes na Região Oeste de Santa Maria/ RS, assim como investigar a incidência de quedas, ao mesmo tempo em que se buscou desenvolver estratégias para a prevenção de quedas em idosos. A pesquisa foi do tipo descritivo-qualitativa. A amostra foi composta por 20 idosos, de ambos os sexos, com idade média de 75 anos. Os instrumentos da pesquisa foram o MiniExame do Estado Mental (MEEM), Escala de Tinneti para avaliar o equilíbrio e a marcha, e também realizaram-se testes de força dos principais grupos musculares envolvidos na instabilidade postural. Realizou-se ainda avaliação das alterações visuais e vestibulopatias referidas e observação do ambiente domiciliar para risco de quedas. A incidência de quedas recorrentes foi de $75 \%$ dos idosos investigados e os resultados encontrados indicam que a queda está associada ao cognitivo, alterações visuais, vestibulopatias e ao ambiente domiciliar. Fatores isolados que não apresentaram riscos foram o equilíbrio, a marcha e a força muscular.

\section{Abstract}

Physiotherapy plays an important role in preventing falls in the elderly. This study aimed to investigate the risk factors that predispose to falls in elderly residents of the West of Santa Maria / RS, as well as to investigate the incidence of falls at the same time, seeking to develop strategies for the prevention of falls in the elderly. The research was descriptive and qualitative. The sample consisted of 20 elderly of both sexes, with an average age of 75 years. The instruments of research were the Mini-Mental State Examination (MMSE), Tinneti scale to assess the balance and gait and also took tests of strength are the major muscle groups involved in postural instability. We also assessed the visual changes and dizzy

\footnotetext{
Centro Universitário Franciscano, Área de Ciências da Saúde, Curso de Fisioterapia.

Rua dos Andradas, 1614 - Centro

97010-032 - Santa Maria, RS, Brasil
}

Correspondênca / Correspondence

Ana Carla Piovesan

E-mail: aninhapiovesan@yahoo.com.br

Palavras-chaves: Acidentes por Quedas. Idosos. Fisioterapia. Fatores de Risco. 
referred to the observation of households concerning the risk of falls. The incidence of recurrent falls was $75 \%$ of elderly investigated and the results show that falls are associated with cognitive, visual changes, dizzy and the home environment. Factors that alone did not show the risks were balance, running and muscle strength.
Key words: Falls. Elderly. Physiotherapy. Risk Factors.

\section{INTRODUÇÃO}

O envelhecimento, antes considerado um fenômeno, hoje faz parte da realidade da maioria das sociedades. O mundo está envelhecendo. Tanto isso é verdade que se estima para o ano de 2050, que existam cerca de dois bilhões de pessoas com 60 anos ou mais no mundo, a maioria vivendo em países em desenvolvimento. No Brasil, estima-se que existam, atualmente, cerca de 17,6 milhões de idosos. ${ }^{1}$

É muito comum no envelhecimento acontecerem alterações progressivas no organismo, tornando-o mais suscetível às agressões intrínsecas ou extrínsecas. Um exemplo, dentro desta suscetibilidade é a queda, que pode estar relacionada a instabilidade postural e alterações dos sistemas sensoriais e motor. ${ }^{2}$

Sabe-se que o desenvolvimento das alterações devidas ao envelhecimento segue um padrão que é exclusivo de cada indivíduo, as quais se processam à medida que a idade avança. Tal fator faz repensar as repercussões da idade sobre a função, que podem levar à queda da pessoa idosa, entre as quais se observam: diminuição da força muscular, dos reflexos, da flexibilidade, da velocidade espontânea da marcha, acuidade visual e função vestibular. ${ }^{3}$

Nessa direção, observa-se que as quedas são causas importantes de morbidade entre os idosos e podem ter consequências desastrosas. Além do risco de fraturas, há perda de confiança para caminhar, devido ao temor de novas quedas, fazendo o idoso diminuir sua mobilidade, formando-se um círculo vicioso, pois com a restrição de atividades há diminuição da força muscular, enfraquecimento dos membros inferiores, levando à condição de dependência e isolamento social. ${ }^{4}$
Segundo o Ministério da Saúde (MS) cerca de $30 \%$ das pessoas idosas caem a cada ano. Essa taxa aumenta para $40 \%$ entre os idosos com mais de 80 anos e $50 \%$ entre os idosos institucionalizados. As mulheres tendem a cair mais que os homens até os 75 anos de idade, a partir dessa faixa etária as frequências se igualam. Dos que caem, cerca de $25 \%$ requerem hospitalização e, destes, apenas metade sobreviverá após um ano. ${ }^{1}$

Nesse sentido, a fisioterapia surge como uma área de conhecimento que desperta seu olhar para a saúde do idoso, buscando manter e melhorar a funcionalidade, com vistas à autonomia do sujeito e a qualidade de vida. A fisioterapia desempenha importante papel na prevenção das quedas, pois proporciona melhora do quadro motor e do equilíbrio, além de fornecer orientações aos pacientes e seus cuidadores, eliminando ou minimizando os fatores de risco.

Considerando o exposto e que as quedas, ao mesmo tempo em que comprometem a qualidade de vida dos idosos, custam caro aos serviços de saúde, pois exigem a internação do paciente, fazse necessário identificar as causas de quedas em idosos, despertando o olhar dos profissionais da saúde para a prevenção das mesmas. Sendo assim, esta pesquisa teve como objetivos investigar os fatores de risco que predispõem a quedas em idosos residentes na Região Oeste de Santa Maria/ RS, assim como investigar a incidência de quedas, ao mesmo tempo em que se buscou desenvolver estratégias para a prevenção de quedas em idosos.

\section{METODOLOGIA}

O presente trabalho caracterizou-se como um estudo qualitativo do tipo descritivo, visto que a 
análise qualitativa "incorpora a questão do significado e da intencionalidade como inerentes aos atos, às relações, e às estruturas sociais, como construções humanas significativas" ${ }^{5}$

Este estudo apresentou também uma abordagem descritiva. Segundo Michel, a pesquisa descritiva tem o propósito de analisar fatos ou fenômenos em sua natureza e características, procurando observar, registrar e analisar suas relações, conexões e interferências. ${ }^{6}$

Respeitando os aspectos éticos com seres humanos, esta pesquisa foi submetida e aprovada pelo Comitê de Ética em Pesquisa da UNIFRA, sob n ${ }^{\circ}$ 092.2008-2. Também foi solicitada autorização junto à Secretaria Municipal de Saúde e à Unidade de Estratégia de Saúde da Família Roberto Binato (UESF). Os participantes da pesquisa foram convidados a assinar o termo de consentimento livre e esclarecido, concordando com a participação.

A amostra foi composta por 20 idosos, indicados pela equipe da UESF (Unidade Estratégia de Saúde da Família Roberto Binato), residentes na região Oeste de Santa Maria/RS e pertencentes à área de abrangência da referida unidade. Foram adotados como critérios de exclusão idosos acamados que não deambulam e/ou com déficit cognitivo que impossibilitasse a realização do questionário e/ou dos testes.

A coleta dos dados foi realizada no período de agosto a setembro de 2008, através de uma visita domiciliar em cada residência dos participantes, acompanhadas dos agentes comunitários de saúde (ACS), utilizando-se quatro instrumentos de avaliação e um diário de campo.?

Para avaliação cognitiva, foi realizado o MiniExame do Estado Mental (MEEM), que auxilia na identificação das principais alterações na saúde mental das pessoas idosas, visto que possui como escore um total de 35 pontos. A avaliação do equilíbrio e marcha foi realizada através da Escala de Tinneti, segundo $\mathrm{MS}^{1}$, cujo escore total é de 28 pontos.
A avaliação da força muscular realizou-se conforme Palmer \& Epler ${ }^{8}$, sendo que os grupos musculares alvos deste estudo foram: flexores plantares, tibial anterior, quadríceps abdutores de quadril, isquiostibiais, glúteos, abdominais e paravertebrais.

Também foram realizadas as avaliações das alterações visuais e auditivas referidas, onde se verificaram o uso de órteses visuais e auditivas, além de sinais de vestibulopatias referidas. ${ }^{9}$

A análise do ambiente domiciliar do idoso foi obtida através da identificação dos fatores de risco para as quedas (iluminação inadequada, superfícies escorregadias, tapetes soltos ou com dobras, degraus altos ou estreitos, obstáculos no caminho - móveis baixos, pequenos objetos e fios -, ausência de barra de apoio em corredores, escadas e banheiros, prateleiras excessivamente baixas ou elevadas ou calçados inadequados) (MS). ${ }^{1}$ Essas informações foram registradas no diário de campo. Também foi registrada nesse instrumento a história de quedas anteriores do idoso, assim como as repercussões causadas por estas.

A partir dos resultados da avaliação do ambiente domiciliar, desenvolveu-se uma cartilha com orientações para prevenção de quedas, que foi entregue aos participantes como retorno da pesquisa.

Os dados foram analisados qualitativamente de acordo com a frequência das informações contidas no diário de campo, e quantitativamente através dos testes aplicados, sendo apresentadas através da estatística descritiva, expressas em percentuais, utilizando-se figuras e quadros.

\section{ANÁLISE E DISCUSSÃO DOS DADOS}

Os sujeitos de pesquisa foram 20 idosos com idades entre 60 e 90 anos com média de 75 anos, sendo 18 mulheres e dois homens. Dos idosos investigados, 15 possuem histórias de quedas recorrentes, representando $75 \%$ da amostra, mas nenhuma das quedas provocou fratura ou lesões mais graves. 
A avaliação cognitiva realizada através do MEEM, possui uma pontuação total com escore de 35 pontos e classifica em nível de escolaridade através do escore alcançado. O MEEM mostrou que, dos 20 idosos avaliados, seis tiveram escores menores que 19 (pontuação de 13 a 18), representando $30 \%$ da amostra, sendo classificados como analfabetos. Outros cinco idosos tiveram escores de 19 a 23 pontos, representando $25 \%$ da amostra, sendo classificados como 1 a 3 anos de escolaridade. Escores de 24 a 28 pontos, ocorreram em outros cinco idosos representando $25 \%$ da amostra, sendo classificados de 4 a 7 anos de escolaridade. Dos 20 idosos, apenas quatro idosos alcançaram mais de 28 pontos, representando $20 \%$ da amostra, classificados como tendo mais que 7 anos de escolaridade. Como a habilidade de cálculo e a perda da memória recente são indicadores da redução das funções cognitivas, o MEEM avalia o estado cognitivo classificando em grau de escolaridade. A figura 1 mostra os resultados da avaliação cognitiva, relacionando o número de participantes com o nível de escolaridade.

Figura 1 - Avaliação da função cognitiva através do Mini-Exame do Estado Mental.

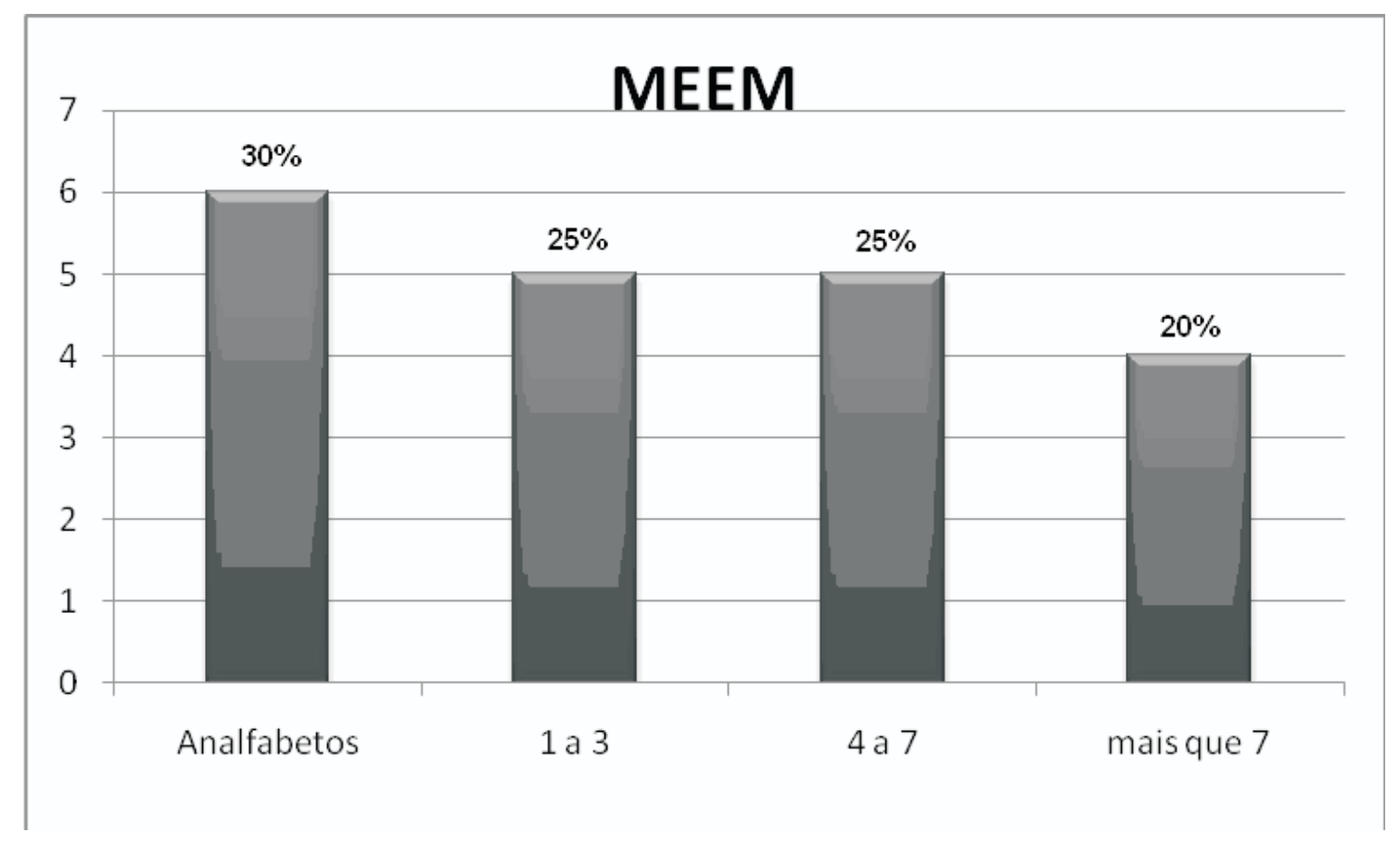

Conforme os resultados encontrados na avaliação do MEEM entre os idosos pesquisados, a figura 1 mostra que o cognitivo no idoso predispõe a risco de quedas. Nesse sentido, de acordo com Abrams \& Berkow, ${ }^{10}$ a frequência de quedas aumenta devido à falta de percepção de perigos ambientais e das próprias capacidades mentais dos idosos.

Já os estudos de Aragão \& Navarro ${ }^{11}$ indicam que existe uma relação direta entre a progressão da idade e o aumento do comprometimento cognitivo dos idosos, mas seus resultados não indicam correlação entre as alterações cognitivas e aumento do índice de quedas.

Luzio et al. ${ }^{12}$ registram que a coordenação motora e a concentração estão diretamente influenciadas pelo cognitivo, traduzindo-se na dificuldade em realizar simultaneamente duas ou mais tarefas - por exemplo, conversar e caminhar, o que predispõe a quedas. Segundo o MS ${ }^{1}$, o desempenho físico e social do idoso depende da integridade de suas funções cognitivas. 
Para a avaliação do equilíbrio e da marcha, utilizou-se a Escala de Tinneti, que possui uma pontuação variando de 0 a 28 pontos. Quanto menor a pontuação, maior o comprometimento do equilíbrio e da marcha. Pontuação menor que 19 pontos indica um aumento no risco de quedas de cinco vezes. ${ }^{1}$ De acordo com a análise dos dados, os escores apresentados variaram de $21 \mathrm{a}$ 28 pontos, com média de 24 pontos. A figura 2 apresenta a avaliação do equilíbrio e marcha, demonstrando o número de idosos com suas respectivas pontuações.

Figura 2- Avaliação do equilíbrio e marcha através da Escala de Tinneti.

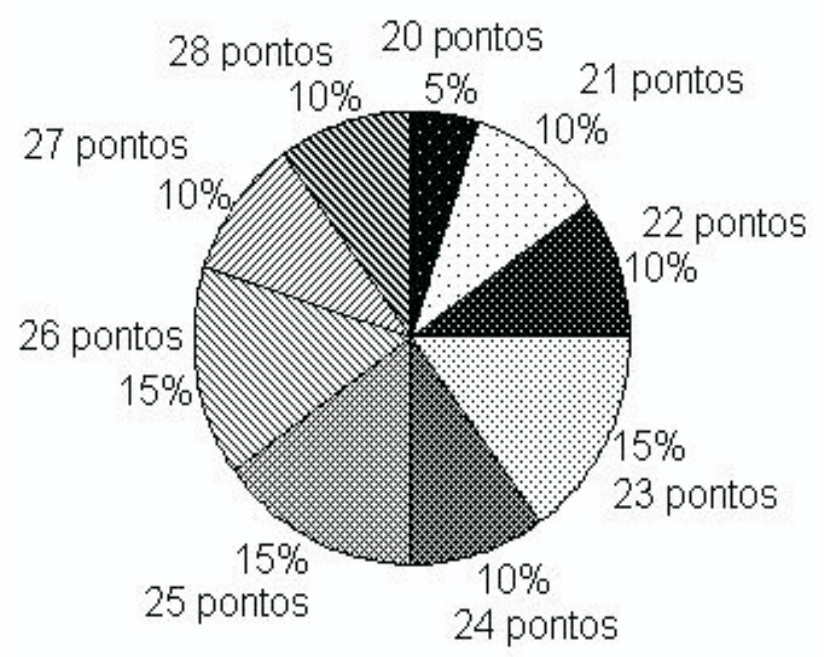

Os dados desta pesquisa revelam que nenhum idoso apresentou escore menor que 19 pontos, o que significaria risco cinco vezes maior de quedas. Além disso, eles revelam que não existe risco de quedas para esse grupo de idosos, considerando os fatores de equilíbrio e marcha. No entanto, não se pode afirmar que não há ausência de risco, pois $75 \%$ dos idosos apresentaram episódios de quedas recorrentes, mas esses episódios de quedas podem estar relacionados a outros fatores.

Segundo Guimarães \& Farinatti, ${ }^{13}$ as alterações do equilíbrio na população idosa são problemas relativamente comuns. Estes levam a importantes limitações na realização das atividades de vida diária (AVDs), sendo, portanto, uma das principais causas de quedas.

Segundo o Ministério da Saúde, a grande propensão da pessoa idosa à instabilidade postural e à alteração da marcha aumenta o risco de quedas. As alterações na mobilidade e quedas podem ocorrer por disfunções motoras, de sensopercepção, equilíbrio ou déficit cognitivo. A dinâmica do aparelho locomotor sofre alterações com uma redução na amplitude de movimentos, tendendo a modificar a marcha, realizando passos mais curtos e mais lentos, com tendência a arrastar os pés. A amplitude de movimento dos braços também diminui, ficando estes mais próximos do corpo. A base de sustentação se amplia e o centro de gravidade corporal tende a se adiantar, em busca de maior equilíbrio. ${ }^{1}$

A avaliação da força muscular foi realizada nos grupos musculares descritos, conforme apresentado no quadro 1 , sendo obedecidos os seguintes critérios de classificação: grau 5 (normal), grau 4 (bom), grau 3 (regular), grau 2 (fraco), grau 1 (esboço), grau 0 (nulo). 
Quadro 1 - Grau de força muscular dos idosos.

Avaliação da força muscular

\begin{tabular}{|c|c|c|c|c|c|c|c|}
\hline Músculos & Grau5 & Grau4 & Grau3 & Grau2 & Grau1 & Grau0 & $\begin{array}{c}\text { Total } \\
\text { de } \\
\text { idosos }\end{array}$ \\
\hline $\begin{array}{c}\text { Flexores } \\
\text { Plantares }\end{array}$ & 19 & 1 & - & - & - & - & 20 \\
\hline Tibial anterior & 19 & 1 & - & - & - & - & 20 \\
\hline Quadríceps & 18 & 2 & - & - & - & - & 20 \\
\hline Abdutores do quadril & 20 & - & - & - & - & - & 20 \\
\hline Isquiostibiais & 13 & 3 & 4 & - & - & - & 20 \\
\hline Glúteos & 16 & 3 & 1 & - & - & - & 20 \\
\hline Paravertebrais & 10 & 5 & 2 & 2 & - & - & 19 \\
\hline Abdominais & 9 & 5 & 3 & 1 & 1 & - & 19 \\
\hline
\end{tabular}

De acordo com os resultados encontrados, dois idosos apresentaram força grau $5 \mathrm{em}$ todos os grupos musculares. Da mesma maneira, todos os idosos apresentaram força grau 5 em abdutores de quadril, sendo que a maior variação dos critérios de classificação ocorreu em abdominais e paravertebrais. Destaca-se que não foi possível realizar teste de força abdominal e paravertebral, por incapacidade de um usuário no momento da avaliação, o que explica o número total de idosos igual a 19 no quadro 1.

Os resultados encontrados na pesquisa não evidenciaram risco de quedas, conforme a avaliação da força dos grupos musculares flexores plantares, tibial anterior, quadríceps e abdutores do quadril desses idosos, pois não houve grandes variações nos critérios de avaliação, variando entre grau 5 e grau 4 de força muscular. Porém, quanto à estabilidade postural, pode-se considerar que poderia haver possibilidade de quedas devido à variação nos critérios de avaliação em isquiostibiais, glúteos, abdominais e paravertebrais, tendo uma variação de força muscular entre grau 5 e grau 1.

De acordo com Pickles et al., ${ }^{3}$ a fraqueza dos membros inferiores favorece as quedas, como acontece na pessoa que é incapaz de fazer um traslado bem-sucedido ou que não consegue ficar em pé por tempo suficiente, sem segurar-se em alguma coisa, ao se vestir, após usar o banheiro.

Segundo Paixão Junior \& Heckmann, ${ }^{14}$ para manter uma postura ereta e estável sob condições estáticas, é necessária a ativação muscular coordenada para manter o corpo ereto nas AVDs. Um grupo muscular apresentando déficit de força predispõe a instabilidade postural e perda de equilíbrio, consequentemente levando às quedas.

A avaliação do sistema visual referida também se reportou à análise de vestibulopatias. Dos 20 idosos avaliados, 18 possuem alterações visuais, representando $90 \%$ da amostra, sendo que 13 apresentaram alterações visuais e usam órteses, dois não apresentaram alterações visuais e não usam órteses e cinco apresentaram alterações visuais e não usam órteses. $\mathrm{O}$ quadro 2 mostra esses resultados com seus respectivos percentuais. A partir desses resultados, a visão constitui um fator de risco para as quedas, pois $90 \%$ da amostra apresentaram alterações visuais, predispondo episódios de quedas recorrentes. 
Quadro 2 - Número de idosos com alterações visuais e sua relação com o uso de órteses visuais.

Avaliação das alterações visuais referidas

\begin{tabular}{|c|c|c|}
\hline & Número de idosos & Percentual \\
\hline $\begin{array}{c}\text { Idosos com alteraçōes na visão que } \\
\text { usam órteses }\end{array}$ & 13 & $65 \%$ \\
\hline Idosos sem alterações na visão & 2 & $10 \%$ \\
\hline $\begin{array}{c}\text { Idosos com alteraçós na visão que } \\
\text { não usam órteses }\end{array}$ & 5 & $25 \%$ \\
\hline
\end{tabular}

Rozenfeld ${ }^{15}$ e Vernon ${ }^{16}$ corroboram este estudo, pois revelam, da mesma forma, que a incidência de quedas nos idosos ocorre devido às alterações visuais ocorridas no envelhecimento.

Da mesma forma, Perracini \& $\operatorname{Ramos}^{17}$, em estudo realizado com idosos do município de São Paulo, constataram que a visão deficiente é um dos fatores de risco para quedas.

Segundo Vernon ${ }^{16}$, a visão é também um importante fator no equilíbrio e na marcha, interagindo intimamente com a função cognitiva, podendo ser responsáveis por até $20 \%$ da taxa de acidentes em quedas.

$\mathrm{Na}$ idade avançada, há incidência elevada de doenças como catarata, glaucoma e retinopatia, que podem comprometer a capacidade de julgar uma queda iminente e proceder à ação corretiva. A interpretação da informação espacial pode estar comprometida por déficit na acuidade visual, restrição do campo visual, aumento da suscetibilidade à luz, percepção de profundidade deficiente ou instabilidade na fixação do olhar. ${ }^{15}$

Do total da amostra avaliada, 17 idosos também apresentaram sinais de vestibulopatias, sendo que as mais relatadas foram: tontura (75\%), cefaleia (60\%), taquicardia (45\%) e ansiedade $(45 \%)$. Os resultados de vestibulopatias predispõem riscos de quedas isolados ou associados com outros fatores de risco, como por exemplo, a visão.

Para Herdman, ${ }^{18}$ a tontura de origem vestibular pode ser um fator desencadeante de quedas recorrentes, pois as disfunções vestibulares limitam o controle postural, predispondo à instabilidade e ao desalinhamento corporal.

De acordo com Abrams \& Berkow ${ }^{10}$, os sintomas de tontura ou falta de equilíbrio podem, às vezes, ser desencadeados por extensão da cabeça, como por exemplo, quando o indivíduo procura pegar alguma coisa em uma estante alta. Parker ${ }^{19}$ explica que o vestíbulo responde principalmente à posição da cabeça em relação à gravidade (equilíbrio estático), enquanto os canais semicirculares do ouvido interno reagem principalmente à velocidade e direção dos movimentos da cabeça (equilíbrio dinâmico). $\mathrm{Na}$ prática, todos eles respondem à maioria das posições e dos movimentos da cabeça.

Parker ${ }^{19}$ também afirma que a cefaleia está frequentemente associada a distúrbios visuais, náuseas e vômitos. A tontura pode resultar de um distúrbio que afeta os órgãos do equilíbrio, podendo impossibilitar a pessoa de caminhar ou ficar em pé, o que pode levá-la à queda.

$\mathrm{Na}$ avaliação do ambiente domiciliar, foram identificados como riscos de quedas mais frequentes: ausência de barra no banheiro, presença de tapetes, presença de degraus, tapete sem antiderrapante no banheiro e acesso difícil ao interruptor de luz. Poucos dispositivos de segurança foram encontrados no banheiro, como, por exemplo, barras de apoio, apesar de terem sido encontrados idosos com incapacidades e necessidades desses dispositivos de segurança. A figura 3 representa os riscos ambientais encontrados através da avaliação. 
Figura 3 - Avaliação do ambiente domiciliar. Principais riscos ambientais encontrados nas residências dos idosos.

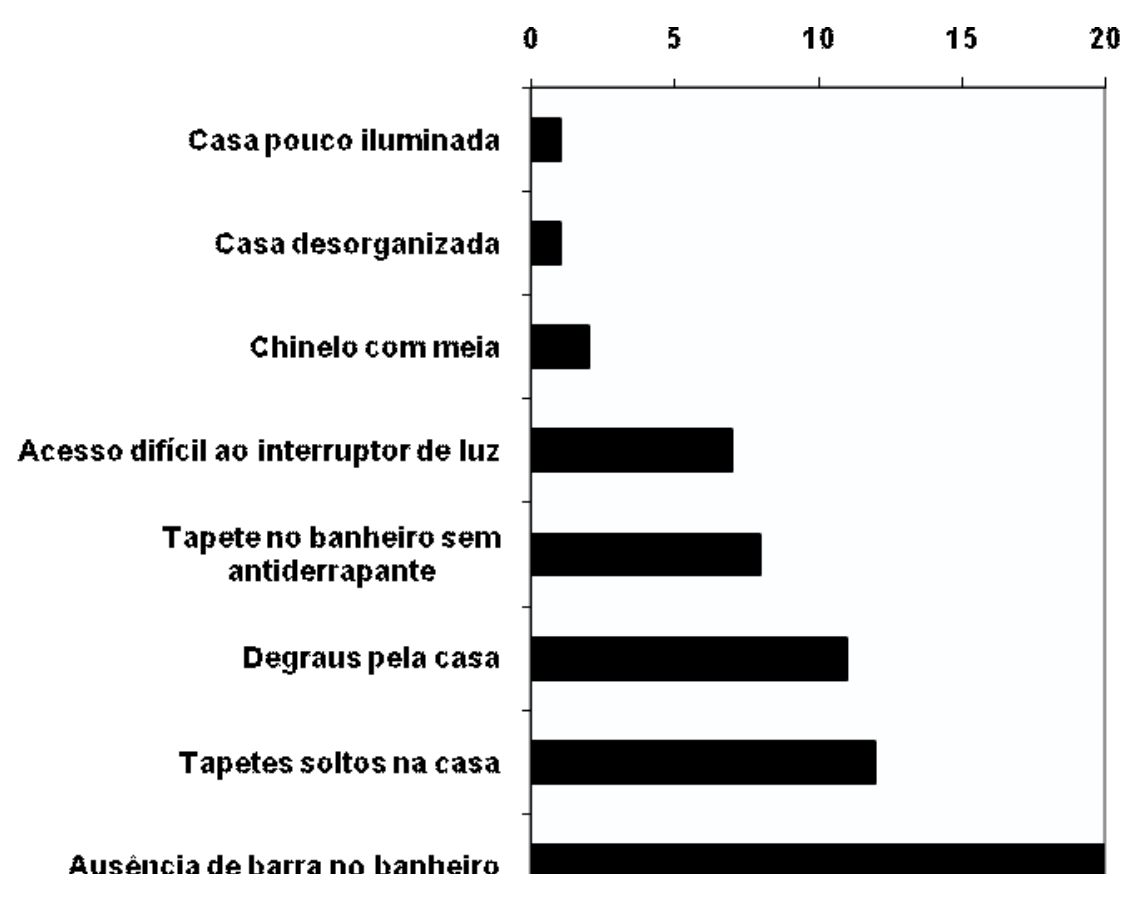

Quanto à avaliação ambiental, percebe-se uma alta prevalência de riscos na população estudada. Nenhum domićlio avaliado esteve livre de riscos ambientais, o que se torna um fator de risco importante para as quedas.

Segundo o MS ${ }^{1}$, a maioria das quedas acidentais ocorre dentro de casa ou em seus arredores, geralmente durante o desempenho de atividades cotidianas como caminhar, mudar de posição e ir ao banheiro. A influência dos fatores ambientais no risco de quedas associa-se ao estado funcional e mobilidade da pessoa idosa. Quanto mais frágil o idoso, mais suscetível a quedas. Manobras posturais e obstáculos ambientais que não são problemas para idosos mais saudáveis podem, no entanto, transformar-se em séria ameaça à segurança e mobilidade daqueles com alterações no equilíbrio e marcha.

Ferrer et al. apud Clemson Cumming ${ }^{20}$ só encontraram prevalência maior de riscos ambientais presentes na casa de idosos que caíram quando estes apresentavam déficit cognitivo, atribuindo isso ao fato de que riscos ambientais isolados não teriam força suficiente para levar um idoso à queda. Também relatam que as características intrínsecas do idoso, somadas aos vários fatores ambientais que ultrapassem sua capacidade físico-funcional, resultariam em evento de queda.

\section{CONCLUSÃO}

Como visto, as quedas ocorrem pela combinação de vários fatores. Para que se possa atuar, modificando o que for necessário, é importante a identificação destes no contexto de vida do idoso. A amostra estudada evidenciou que o risco de quedas está associado às alterações visuais referidas, alterações no cognitivo, sinais de vestibulopatias referidas e aos fatores de risco do ambiente domiciliar. Os resultados das avaliações da força e do equilíbrio nos idosos não evidenciaram risco para as quedas no grupo pesquisado.

Com o aumento da expectativa de vida, há uma preocupação em conseguir contribuir para a 
qualidade de vida da população idosa, prevenindo as doenças crônicas nãotransmissíveis, apesar das progressivas limitações que possam ocorrer. Para tanto, é necessário que sejam desenvolvidos mais estudos sobre a pessoa, produzindo indicadores de saúde para que se possa planejar ações multiprofissionais, estabelecendo redes, desvelando as particularidades dessa população, assim como os riscos que predispõem a quedas, pois estas elevam os índices de morbidade no idoso.

\section{REFERÊNCIAS}

1. Ministério da saúde (Brasil). Envelhecimento e saúde da pessoa idosa Caderno de Atenção Básica 2006 ; (19)

2. Guimarães LHCT, Galdino DCA, Martins FLM, Vitorino DFM, Pereira KL, Carvalho EM. Comparação da propensão de quedas entre idosos que praticam atividade física e os idosos sedentários. Rev Neurociências 2004; 12 (2).

3. Pickles B, Compton A, Cott C, Simpson J, Vandervoort A. Fisioterapia na terceira idade. $2^{\circ}$ ed. São Paulo: Santos; 2000.

4. Yuaso DR, Sguizzatto GT. Fisioterapia em pacientes idosos. In: Netto MP. Gerontologia: a velhice e o envelhecimento em visão globalizada. São Paulo: Atheneu; 1999.

5. Oliveira SL. Tratado de Metodologia Científica: projetos de pesquisas TGI, TCC, monografias, dissertações e teses. São Paulo: Pioneira; 2001.

6. Michel MH. Metodologia e pesquisa científica em ciências sociais. São Paulo, SP: Atlas;2005.

7. Triviños ANS. Introdução à pesquisa em ciências sociais: a pesquisa qualitativa em educação: o positivismo; a fenomenologia; o marxismo. São Paulo: Atlas; 1987.

8. Palmer ML, Epler ME. Fundamentos das técnicas de avaliação musculoesquelética. 2 ed. Rio de Janeiro: Guanabara Koogan ; 2000.

9. Caixeta R. Instabilidade postural e quedas no idoso. In: Hargreaves, LHH. Geriatria. Brasília: Editora Secretaria Especial de editoração e publicações do Senado Federal; 2006: p. 467 - 486.

10. Abrams WB, Berkow R. Manual Merck de Geriatria: 7 ed. São Paulo: Roca;1995.

11. Aragão FA, Navarro FM. Influências do envelhecimento, do tempo de evolução da doença e do estado cognitivo sobre os episódios de quedas, em uma população parkinsoniana. Rev. Fisioterapia Brasil. 2008: v. 06, nº 4, 250254, Julho/Agosto.
12. Luzio CS, Garcia FV, Benzinho T, Veiga VG. Programa de Prevenção de Quedas no Idoso. In: XXX Congresso do NES, realizado no Porto 2003.

13. Guimarães JMN, Farinatti PTV. Análise descritiva de variáveis teoricamente associadas ao risco de quedas em mulheres idosas. Rev. Bras. Med. Esporte. 2005 set/Out;11(5): 299.

14. Paixão Junior CM, Heckmann MF. Distúrbios da postura, marcha e quedas. In: Freitas EV, Py L, Néri AL, Cançado FAX, Gorzoni ML, Rocha SM. Tratado de Geriatria e Gerontologia. Rio de Janeiro :Guanabara Koogan 2002; 624-634.

15. Rozenfeld S. Reações Adversas aos Medicamentos na Terceira Idade: as Quedas Como Iatrogenia Farmacoterapêutica 1997.Rio de Janeiro .Tese. [Doutorado em Medicina Social] -Universidade do Estado do Rio de Janeiro; 1997.

16. Vernon MS. Reichel Assistência ao idoso. In: Gallo JJ, Busby - Whitehead J, Rabins PV, Silliman RA, Murphy JB. Aspectos clínicos do envelhecimento. 5.ed.Rio de Janeiro: Guanabara Koogan; 2001.

17. Perracini MR, Ramos LR. Fatores associados a quedas em uma coorte de idosos residentes na comunidade. Rev. Saúde Pública 2002; 36: 709-16.

18. Herdman SJ. Reabilitação Vestibular. São Paulo: Manole; 2002.

19. Parker S. O livro do corpo humano. São Paulo: Ciranda Cultural; 2007.

20. Ferrer MLP, Perracini MR, Ramos LR. Prevalência de fatores ambientais associados a quedas em idosos residentes na comunidade em São Paulo, SP. in: Clemson L, Cumming M. Case-control study of hazards in the home and risk of falls and hip fractures.Age Ageing. 1996; 25: 97-101. 
